\title{
Courage, collaboration, complexité et innocuité de la chimiothérapie: une vue depuis la ligne de feu
}

par Esther Green, Rachel White, Karen Janes, Anthony Fields et Anthony Easty

\section{Abrégé}

La communauté canadienne de l'oncologie a été dévastée en août 2006 par l'annonce du décès d'une patiente d'une surdose de fluorouracile. Alors que nous pensions que nos mécanismes de régulation garantissaient la sécurité des patients, nous savions dorénavant qu'ils ne suffisaient pas. La pratique a immédiatement commencé à changer dans l'ensemble du pays. Cependant, le compte rendu d'incident soulignait qu'il y avait encore bien des choses que nous ignorions encore à propos des enjeux de sécurité pour le patient relativement à l'administration de la chimiothérapie IV ambulatoire au Canada. Pour y répondre, une équipe interdisciplinaire pancanadienne a lancé une étude exploratoire de 18 mois laquelle a produit un rapport dégageant plusieurs enjeux de sécurité et les recommandations afférentes. Le présent article résume les aperçus clés que nous avons rassemblés pour les infirmières en oncologie du Canada dans le cadre de notre participation à cette étude : qu'il nous faut du courage pour nous exprimer et divulguer les préoccupations en matière d'innocuité; qu'il nous faut collaborer afin de trouver des améliorations qui fonctionnent pour tout le monde; et que nous devons nous efforcer de simplifier notre travail à la ligne de feu en réduisant la complexité en amont et dans l'ensemble du système.

\section{Contexte}

La communauté canadienne de l'oncologie a été dévastée en août 2006 par l'annonce du décès d'une patiente d'une surdose de fluo-

\section{Au sujet des auteurs}

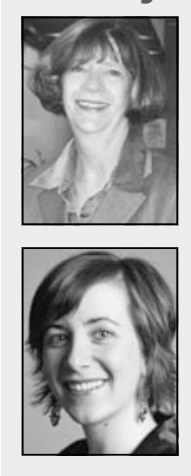

Esther Green, B.Sc.inf., M.Sc.(T), Provincial Head Nursing and Psychosocial Oncology, Action cancer Ontario

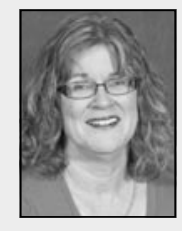

Rachel White, MA, Health Technology Safety Research Team, Réseau universitaire de santé

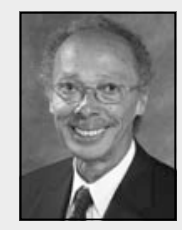

Karen Janes, B.Sc.inf., M.Sc.inf., Nursing Practice Leader at the BC Cancer Agency

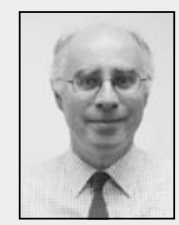

Anthony Fields, MA, MD, L.M.C.C., R.C.P.S.C., Vice President, Cancer Corridor, Alberta Health Services and Professor of Oncology at the University of Alberta

Anthony Easty, Ph.D., P.Eng., CCE, Health Technology Safety Research Team, Réseau universitaire de santé, Institute of Biomaterials and Biomedical Engineering, Université de Toronto rouracile au Cross Cancer Institute (CCI) situé à Edmonton, Alberta. La pompe de perfusion de Denise Melanson a été accidentellement programmée pour administrer le médicament en l'espace de quatre heures plutôt que de quatre jours. Quelques mois après, l'enquête sur l'incident (ISMP Canada, 2007) a révélé que plusieurs autres décès similaires liés à l'administration de chimiothérapie avaient récemment eu lieu en Amérique du Nord, soulignant ainsi qu'il ne s'agissait pas d'un incident isolé. Alors que nous pensions que nos mécanismes de régulation garantissaient la sécurité des patients, nous savions dorénavant qu'ils ne suffisaient pas.

L'analyse des causes fondamentales (ACF) de l'incident de surdose de fluorouracile par l'Institut pour l'utilisation sécuritaire des médicaments (IUSM) du Canada a cerné 16 facteurs de causalité et a élaboré plusieurs recommandations afférentes en matière de sécurité. L'ACF a été affichée sur Internet afin qu'elle soit largement diffusée. Les pratiques ont immédiatement commencé à changer dans l'ensemble du pays à la lumière de ces recommandations. Toutefois, le rapport soulignait qu'il y avait encore beaucoup de choses que nous ignorions relativement aux enjeux de sécurité pour le patient relativement à la chimiothérapie par intraveineuse (IV) ambulatoire au Canada. Ainsi, l'Association canadienne des agences provinciales de lutte contre le cancer (ACAPLC) a mis sur pied le Comité de l'innocuité des traitements systémiques afin de poursuivre les travaux sur cette question des plus importantes. C'est sous la direction de ce comité qu'une équipe interdisciplinaire et interprovinciale a présenté une demande de subvention auprès de l'Institut canadien pour la sécurité des patients dans le cadre de son concours de projets de recherche 2007. Plusieurs provinces ont contribué des ressources en espèces et en nature. L'étude a reçu un financement et s'est déroulée sur une période de 18 mois.

Le présent article résume les aperçus clés que nous avons rassemblés pour les infirmières en oncologie du Canada dans le cadre de notre participation à cette étude. Ceux-ci ont trait: au courage, à la collaboration et à la complexité.

\section{L'étude}

Cette section résume les objectifs, les méthodes, les résultats et les recommandations liés à l'étude.

\section{Objectifs}

Cette étude de recherche exploratoire visait à :

1. dégager les pratiques actuelles pour commander, préparer, étiqueter, vérifier et administrer la chimiothérapie par voie intraveineuse en contexte de soins ambulatoires au Canada;

2. définir les sources de risque additionnel dans une vaste gamme de contextes de prestation de la chimiothérapie par voie intraveineuse;

3. recommander les stratégies à adopter pour contrer les risques.

\section{Méthodologie}

On a fait appel à plusieurs méthodologies pour déterminer et hiérarchiser les problèmes d'innocuité liés à la chimiothérapie IV ambulatoire au Canada: un sondage national auprès des prestataires de soins en oncologie, des études sur le terrain effectuées dans six centres de traitement anticancéreux ainsi qu'une évaluation proactive des risques basée sur le modèle de Rasmussen sur la gestion du risque dans une situation dynamique (Rasmussen, 1997; Vicente \& Christoffersen, 2006) et sur les modalités de défaillance dans la prestation des soins de santé et l'analyse des effets («Healthcare Failure Modes and Effects Analysis» en anglais) (South Dakota Association of Healthcare Organizations 2001; United States Department of Veteran Affairs, 2001). 


\section{Résultats}

$\mathrm{Au}$ total, 331 médecins, pharmaciens en oncologie, infirmières en oncologie et gestionnaires œuvrant dans le domaine des soins aux personnes atteintes de cancer de l'ensemble du Canada ont rempli le questionnaire du sondage. Ils étaient dans leur grande majorité au courant de l'incident d'administration du fluorouracil et du rapport d'analyse de ses causes fondamentales (ISMP Canada, 2007): 95,5\% des répondants avaient conscience de l'incident et $71 \%$ avaient examiné le rapport. En tout, 213 incidents ont été mentionnés par les 331 répondants.

Des divergences entre les sites de l'étude sur le terrain ont été constatées en termes de : technologie, modèles de travail en équipe, complexité des méthodes de travail, modèles de calendriers de traitements et enfin, rôle de l'agence provinciale de lutte contre le cancer. Les effets de la pression exercée dans le but d'accroître l'efficacité étaient ressentis par le personnel de tous les sites, notamment par celui des pharmacies.

Les données recueillies au moyen de l'ensemble des méthodologies ci-dessus ont permis de dégager les enjeux suivants en matière d'innocuité (Tableau 1) :

\section{Recommandations}

Contrairement à la recherche scientifique dans le domaine de la santé et dans ceux de la sécurité des patients hospitalisés et de la pratique, les méthodes classiques d'évaluation quantitative, telles que les essais contrôlés avec répartition aléatoire, sont de peu d'utilité ou sont carrément à proscrire. Les enjeux et les recommandations présentés dans cette section reposent donc sur des observations qualitatives, les commentaires exprimés par les utilisateurs, des consensus entre experts et les pratiques exemplaires décrites dans la documentation scientifique, le cas échéant.

Pour mettre en œuvre les recommandations contenues dans ce rapport, les prestataires de soins devront sans doute faire preuve de créativité et faire des compromis qui tiennent compte des ressources en personnel, de l'espace disponible ou de leurs budgets. Les recommandations doivent donc être adaptées au contexte local.

\section{Tableau 1. Enjeux en matière d'innocuité dégagés et explorés} dans la recherche

\section{Pompes élastomériques à perfusion intraveineuse utilisées en contexte de soins ambulatoires et dispositifs d'accès}

1.1 Pompes élastomériques à perfusion intraveineuse utilisées en contexte de soins ambulatoires et dispositifs d'accès

1.2 Erreurs dans le choix d'une pompe élastomérique à perfusion intraveineuse en contexte de soins ambulatoires

1.3 Soins à domicile et pompes à perfusion intraveineuse

1.4 Dispositifs d'accès utilisés en conjonction avec des pompes élastomériques à perfusion intraveineuse

\section{Ordonnances et étiquetage}

2.1 Modifications des ordonnances (commandes)

2.2 Formulaires préimprimés : réutilisation de formulaires, notes manuscrites, facilité et souplesse d'utilisation

2.3 Étiquetage et erreurs de programmation des pompes à perfusion d'usage général à grand volume

2.4 Ordonnances non structurées

\section{Pratiques pharmaceutiques}

3.1 Organisation du matériel et processus de travail dans les enceintes de biosécurité

3.2 Absence de contre-vérification de la reconstitution

3.3 Exposition à des produits pharmaceutiques dangereux

\section{Autres facteurs}

4.1 Modèles de calendriers de traitements

4.2 Simplification et uniformisation
Pompes élastomériques à perfusion

\section{intraveineuse et dispositifs d'accès}

Les pompes élastomériques à perfusion intraveineuse constituent une façon simple de prévenir les erreurs massives de débit qui peuvent survenir avec les pompes électroniques programmables, mais plusieurs facteurs peuvent entraîner des écarts de débits importants. Il est nécessaire de rehausser l'éducation au personnel et aux patients afin de veiller à ce que le débit réel des pompes soit aussi conforme que possible au débit nominal.

Afin de prévenir les erreurs de sélection des pompes élastomériques à perfusion intraveineuse, lesquelles peuvent entraîner des perfusions trop rapides, il convient de minimiser, par la simplification et l'uniformisation des protocoles, la gamme des modèles de pompes élastomériques à perfusion intraveineuse dans chaque centre de traitement du cancer. Les modèles de pompes élastomériques à perfusion intraveineuse ayant des débits différents doivent être rangés séparément les uns des autres dans les pharmacies afin de prévenir les erreurs de sélection.

\section{Ordonnances et étiquettes}

On considère souvent que le système informatisé de saisie des ordonnances est le moyen le plus efficace et le plus sécuritaire pour commander des médicaments. Les systèmes conçus en tenant compte des besoins en oncologie et dotés de programmes d'aide à la prise de décisions cliniques permettent de réduire le risque de dosage erroné et de calcul erroné de la surface corporelle ou de la dose de médicament. L'adoption efficace de tels systèmes exige toutefois beaucoup de coordination et des ressources considérables, ce qui n'est pas toujours possible à court terme dans les établissements de soins aux personnes atteintes d'un cancer. De nombreux établissements s'efforcent d'adopter des formulaires d'ordonnance préimprimés qui contribueront à éliminer les ordonnances manuscrites, cette étude s'est donc attardée à la conception des formulaires préimprimés.

- Les établissements qui ne sont pas dotés d'un système informatisé de saisie des ordonnances, tout comme ceux qui sont dotés d'un tel système mais qui acceptent encore des ordonnances en format papier, devraient se doter de formulaires préimprimés pour tous les protocoles de traitement utilisés couramment. Il convient d'éviter de recourir à des formulaires d'ordonnance non structurés.

- Il importe de créer des formulaires pré-imprimés, qui tiennent compte des lignes directrices présentées dans le rapport supplémentaire intitulé Developing Ambulatory Chemotherapy Preprinted Orders (Healthcare Human Factors, 2010).

- Pour s'assurer que les versions utilisées sont toujours les plus récentes, les formulaires devraient provenir d'une seule source et pouvoir être téléchargés électroniquement à partir du site Web ou de l'intranet de l'établissement. Les ordonnances devraient pouvoir être imprimées une à une pour chaque patient.

- Pour aider les infirmières qui doivent programmer et vérifier les pompes, le débit (exprimé dans l'unité qui correspond à celle de la pompe, p. ex. ml/h) devrait figurer sur les étiquettes de chimiothérapie produites par la pharmacie ou sur les formulaires d'ordonnance pré-imprimés pour tous les médicaments administrés à l'aide d'une pompe à perfusion à grand volume ou d'une pompe à perfusion utilisée en contexte de soins ambulatoires.

\section{Pratiques pharmaceutiques}

- Afin de prévenir les risques d'erreur au moment de la préparation, une seule préparation de chimiothérapie devrait être présente dans l'enceinte de biosécurité à la fois.

- Pour s'assurer que l'étiquette appropriée est apposée sur le sac qui lui est associé, les étiquettes ou instructions de préparation devraient toujours accompagner les fournitures de préparation ou le produit final qui a été préparé.

- Une contre-vérification (par un second intervenant, idéalement un pharmacien) devrait être faite pour s'assurer que le volume et le 
type appropriés de diluant ont été aspirés dans la seringue aux fins de reconstitution. Cette vérification devrait s'ajouter à celles qui sont déjà faites pour vérifier le volume des médicaments de chimiothérapie après leur reconstitution.

- Des recherches portant sur la qualité du contenu des sacs de chimiothérapie, à l'aide d'une technique telle que la chromatographie liquide à haute performance, s'imposent pour déterminer le taux d'erreurs de préparation de la chimiothérapie dans les pharmacies canadiennes.

\section{Autres enjeux}

- La simplification et l'uniformisation, au degré hiérarchique le plus élevé du système de la santé, des protocoles, des outils de traitement des ordonnances, de la posologie et du déroulement du travail rehausseront l'innocuité et l'efficacité des soins. Une collaboration s'impose entre les organismes de santé provinciaux ainsi que les centres de cancérologie et tous les intervenants qui prodiguent des soins aux personnes atteintes de cancer.

- Comme de nombreux problèmes sont associés au modèle de calendriers de prestation des traitements le jour même, les établissements devraient évaluer l'incidence de l'adoption d'un modèle de prestation des traitements sur plusieurs jours. Ils devraient également déterminer les endroits et les moments convenant à l'adoption d'un tel modèle et chercher à adopter ce modèle de calendrier de prestation des soins pour faire en sorte que :

- le médecin prescripteur ait accès aux résultats des analyses de sang lorsqu'il commande la chimiothérapie, et

- les traitements ont lieu peu après la rédaction des ordonnances de chimiothérapie, mais

- les traitements ont lieu au moins un jour après la rédaction des ordonnances

- afin d'allouer suffisamment de temps à la pharmacie et au personnel infirmier pour se préparer.

- Des politiques et des procédures devraient être mis en place pour déterminer les personnes qui bénéficieraient le plus de traitements prodigués le jour même (p. ex. les patients à mobilité réduite ou ceux qui habitent loin de l'établissement où ils reçoivent leur traitement).

La version complète du rapport et des recommandations peut être téléchargée à l'adresse www.capca.ca.

\section{Leçons à l'intention des infirmières en oncologie}

\section{Courage}

L'équipe du CCI a fait preuve d'un courage exceptionnel lorsqu'elle a décidé de partager la tragédie qui y est survenue en 2006. Sa reconnaissance précoce de la responsabilité de l'établissement et de son engagement envers la modification du système était sans précédent. Ses membres ont pris des risques personnels et professionnels en divulguant l'incident et en alertant par là même la communauté de l'oncologie de l'existence de graves enjeux de sécurité au niveau des traitements systémiques. Lorsque la même erreur de programmation mettant en jeu le même médicament s'est produite dans une autre province quelques mois plus tard, la vie de la personne concernée a été sauvée grâce aux leçons tirées de la mort de Denise Melanson.

Les changements apportés au système des traitements systémiques de chaque province et territoire du Canada sont les conséquences directes des actions du personnel du CCI. Par exemple, il y a eu un abandon généralisé des pompes à perfusion intraveineuse ambulatoire électroniques au profit des pompes élastomériques à perfusion intraveineuse mécaniques, afin d'empêcher les erreurs massives de débit (Figure 1). Nous croyons fermement que ces changements et d'autres ont eu une incidence positive directe sur la sécurité des patients et ont peut-être empêché des patients de subir des préjudices additionnels.

En plus des changements concrets institués dans la pratique du fait de la sensibilisation à l'incident d'administration du fluorouracile, sa divulgation a eu (et continue d'avoir) une incidence significative sur la culture des soins de santé au Canada. Ces dernières années, la communauté chargée de l'innocuité du système de soins a délaissé, pour la gestion des erreurs, son approche axée sur l'intervenant individuel en faveur d'une approche systémique (Reason, 2000). En reconnaissant qu'il n'y avait pas eu de «négligence» dans l'incident mettant en jeu le fluorouracile, et en mettant l'accent sur la nécessité de changer le «système», le CCI a établi un précédent que d'autres organismes pourront suivre lors de la divulgation d'erreurs. Les réponses aux questions du sondage de notre étude illustrent bien ce fait : 271 incidents et accidents évités de justesse en matière de chimiothérapie ont été décrits, et ces divulgations ont joué un rôle central sur le plan de la détermination et de la catégorisation, par l'étude, des enjeux en matière d'innocuité.

En tant que membres de la communauté de l'oncologie, nous vous encourageons à suivre l'exemple du personnel du CCI en divulguant les événements indésirables et les accidents évités de justesse. Nous avons observé, à l'échelle nationale, un virage culturel vers un environnement «sans honte ni blâme». Cette devise sort facilement de nos lèvres, mais il reste un énorme travail à effectuer avant qu'elle ne prenne vraiment racine dans le lieu de travail. Il faut bien du courage pour partager avec autrui les rapports sur nos erreurs et accidents évités de justesse, et nous espérons que ceci vous encouragera à faire de même dans votre pratique, si vous deviez le faire un jour.

\section{Collaboration}

Cette étude est unique en son genre pour l'étendue de la collaboration : entre les diverses autorités et disciplines scientifiques et au niveau des relations clients-fabricants.

\section{Entre diverses autorités}

Du point de vue géographique, cinq agences provinciales co-commanditaires ont collaboré au financement de l'étude et ont offert des sites pour l'étude sur le terrain. Ces derniers ont tous fourni un environnement à la fois ouvert et accueillant pour l'étude et ont collaboré avec les observateurs afin de mettre en évidence les différents modèles de pratique ainsi que les risques perçus pour la sécurité des patients. Il y avait également une représentation pancanadienne au sein du comité directeur du projet (le Comité de l'innocuité des traitements systémiques de l'ACAPC), qui s'est réuni régulièrement tout au long du projet. De plus, l'ACAPC a joué un rôle de premier plan en ce qui concerne les activités d'orientation de la recherche et de transfert des connaissances et a veillé à ce que l'information soit communiquée à chacune des nombreuses parties intéressées. Dans la communauté infirmière, l'ACIO a constitué la voix nationale des soins infirmiers en fournissant son soutien tout au long de l'étude et en se livrant présentement à l'intégration des résultats de recherche dans les Normes nationales d'administration de la chimiothérapie.

\section{Entre diverses disciplines scientifiques}

La collaboration concernait également les disciplines et les domaines de compétence cliniques. En conformité avec la nature interdisciplinaire de l'administration de la chimiothérapie aux patients, les membres de l'équipe de recherche et du comité directeur représentaient les soins infirmiers en oncologie, la pharmacie en oncologie, l'oncologie médicale, l'oncoradiologie et l'administration sanitaire. Nous pensons que cette représentation interdisciplinaire a favorisé 
la découverte d'un plus grand nombre d'enjeux de sécurité pour les patients et la production de recommandations plus vigoureuses tenant compte de tous les aspects du système de chimiothérapie. En outre, étant donné que le rapport comprend des recommandations pour tous les professionnels et qu'il couvre tous les rôles professionnels pertinents, chaque groupe professionnel a la possibilité de tirer des leçons des autres aspects du système auxquels ils ne participent pas habituellement. Par exemple, une des conclusions majeures de l'étude porte sur les pratiques pharmaceutiques relatives au mélange des médicaments. Nous avons découvert que des erreurs de mélange étaient possibles du fait de la manière dont les produits sont organisés dans la zone de mélange et du fait de l'importante pression exercée sur le personnel de pharmacie dans le but d'accroître l'efficacité et du fait des interruptions dont il souffre (Figure 2). Ce résultat nous a permis de mieux comprendre certains des défis confrontant nos collègues et la nécessité dans laquelle nous nous trouvons de limiter les pressions temporelles que nous plaçons sur le personnel de pharmacie. Nous avons également réalisé que tous les intervenants sont à la fois responsables d'interruptions chez autrui et susceptibles d'être interrompus (Westbrook, Woods, Rob, Dunsmuir \& Day,

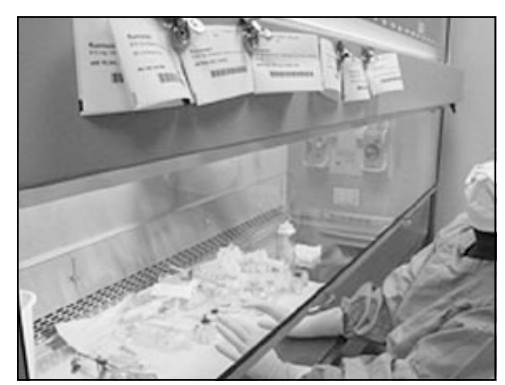

Figure 2. Technicien en pharmacie en train de préparer de multiples mélanges pour différents patients
2010). Les infirmières sont à la fois très présentes dans les services et très accessibles aux patients et aux autres intervenants de santé et elles risquent ainsi d'être visées par davantage d'interruptions et donc de faire davantage d'erreurs. Parfois, nous n'avons aucune conscience de la fréquence des interruptions et de l'incidence de ces dernières ou nous croyons qu'elles font partie intégrante de notre travail.

Les activités de recherche ont principalement été effectuées par des spécialistes des facteurs humains et ce, sous la direction des cliniciens membres de l'équipe. L'ingénierie des facteurs humains ou ergonomie examine les risques au sein d'environnements haute performance en fonction des compétences et des limites connues des intervenants humains, dans la perspective selon laquelle «on ne peut pas changer la condition humaine mais on peut changer les conditions dans lesquelles les humains travaillent (Reason, 2000) [traduction libre]. Ces spécialistes ont contribué des outils systématiques d'analyse ainsi qu'une perspective externe sur les dangers associés à la pratique clinique, et nous estimons que cet engagement a joué un rôle capital dans la réussite du projet. Beaucoup d'infirmières et d'administrateurs de haut niveau ne sont pas au courant de l'existence de l'ingénierie des facteurs humains ni de la façon dont ce domaine spécialisé peut rehausser la pratique clinique. Nous croyons que la formation en facteurs humains devrait faire partie intégrante de la formation infirmière en oncologie et que des spécialistes en facteurs humains devraient jouer un rôle central dans des activités telles que l'évaluation des risques, l'acquisition de nouvelles technologies, la conception d'interfaces-utilisateurs pour le systèmes informatisé de saisie des ordonnances et l'analyse du déroulement des opérations (Cafazzo et al., 2009).

Comme un sous-ensemble du projet se penchait sur la conception des formulaires d'ordonnance pré-imprimés pour la chimiothérapie, l'équipe a également collaboré avec des graphistes en vue de mettre au point des lignes directrices et des modèles téléchargeables. Cette collaboration unique en son genre a approfondi la richesse de la collaboration interdisciplinaire et a permis de produire une directive extrêmement complète et facile à suivre. Nous pensons que pour certaines initiatives concernant la sécurité pour les patients, l'implication de graphistes et de spécialistes des facteurs humains peut déboucher sur d'excellents résultats.

\section{Avec l'industrie}

Le troisième type de collaboration qui est survenu dans le cadre de cette étude a mis en jeu l'équipe de recherche et l'industrie pharmaceutique. Un des thèmes relatifs à l'innocuité cernés par la recherche concernait les pompes élastomériques à perfusion intraveineuse. Beaucoup de centres avaient abandonné ces pompes afin d'éliminer les risques d'erreurs de programmation associés aux pompes électroniques. Selon notre sondage, 96 \% des pompes élastomériques étaient fabriquées par la Baxter Corporation (Baxter Corporation, n.d.). L'équipe de l'étude a contacté les responsables de Baxter Canada (Mississauga, Ontario) qui ont accepté de collaborer avec elle afin de mieux comprendre les facteurs ayant une incidence sur la performance de ces appareils. Nous avons coopéré avec eux en vue d'élaborer un nouveau matériel pédagogique à l'intention des cliniciens et des patients lequel abordait les problèmes cernés dans le cadre de l'étude. Cette expérience nous a appris que lorsque nous approchons un fabricant de manière ouverte et respectueuse, le potentiel de collaboration est élevé, ce qui aboutit à des améliorations aux soins aux patients.

Nous vous encourageons à saisir les opportunités de collaboration avec des collègues au-delà des frontières géographiques et des domaines d'expertise et avec les fournisseurs de technologie. Notre expérience nous montre que de telles collaborations sont extrêmement fructueuses et bénéfiques pour les patients, même si elles peuvent présenter des défis logistiques de taille.

\section{Complexité}

Les traitements systémiques, comme une bonne partie des soins de santé, sont nécessairement complexes. Il existe en effet un vaste nombre de protocoles de traitement systémique, de types, de sièges et de stades de cancer. Chaque protocole se distingue des autres par la combinaison de drogues, la posologie, le calendrier d'administration et les examens exigés. Il est impératif de gérer et d'ajuster attentivement le protocole de chaque malade aux différentes étapes de son traitement. Le système des traitements systémiques doit donc pouvoir composer avec cette complexité afin de traiter les patients en toute efficacité. Cependant, nous avons constaté que certains aspects de cette complexité n'étaient pas requis et qu'il était possible de simplifier le système (Figure 3, Complexité des processus liés à la chimiothérapie, page 84).

Lors de nos visites sur le terrain qui duraient une semaine chacune, nous commencions notre travail en décrivant les processuset en en traçant les grandes lignes-que les membres du personnel suivent depuis le moment où le patient a sa première visite avec l'oncologue jusqu'à ceux où la chimiothérapie est commandée, préparée et administrée. Dans certains sites, nous avons pu saisir rapidement ces processus de première ligne et les décrire facilement au moyen de mots et de diagrammes. En revanche, dans d'autres sites, nous avions besoin de bien plus de temps pour cerner ces processus et dans un cas particulier, il nous a été impossible de dégager les processus dans leur totalité. Bien entendu, nous aurions pu nous en vouloir de ne pas être capables de comprendre les processus, mais lorsque nous avons comparé les pratiques des divers sites, nous avons commencé à nous rendre compte que la question de complexité se rapportait souvent au degré d'uniformisation des échelons hiérarchiques les plus élevés du système. Par exemple, dans un site particulier, les oncologues choisissaient parmi un nombre quasiment illimité de protocoles, utilisaient des blocs d'ordonnances universels non structurés et dictaient les algorithmes en tenant compte de pratiques et de méthodes de communication qu'ils sélectionnaient eux-mêmes. Chaque oncologue avait ainsi une pratique différente. Il s'ensuit que le personnel de première ligne (en pharmacie et soins infirmiers) devait suivre un système extrêmement complexe de processus variables et prendre bien plus de décisions que le personnel d'autres sites. À titre de comparaison, les oncologues d'un autre site disposaient de choix limités sur le plan des 
protocoles à administrer aux patients en fonction des sièges/stades de cancer particuliers. Cette uniformisation se produisait au niveau provincial et l'agence provinciale fournissait également des outils d'ordonnance normalisés (formulaires d'ordonnance pré-imprimés). Cela fait que le personnel en aval devait composer avec moins de variabilité, de points de décision et de complexité, et, conséquemment, moins de confusion. Nous croyons fermement que cet appui à l'uniformisation aboutit à un système plus sûr-bien qu'il exige une coordination importante de la part des administrateurs supérieurs.

Un autre exemple de complexité a directement trait à l'incident au fluorouracile. Lors de la survenue de cet incident, deux types de pompes à perfusion intraveineuse ambulatoire électroniques étaient utilisées dans la région sanitaire concernée. Un type de pompe devait être programmé en ml par heure et un autre en ml par jour. Les étiquettes de médicaments présentaient donc les deux taux de perfusion. Un des changements immédiats apportés à la pratique à la suite de l'incident fut de normaliser les pompes utilisées dans la région sanitaire : les pompes dont le débit était exprimé en ml par jour ont tout de suite été retirées des sites et ce, dans le but de réduire la complexité du système. Il s'agit d'un excellent exemple de ce que nous pouvons faire relativement à bien des aspects des traitements systémiques au Canada, et nous vous invitons vivement à rechercher l'application de ce genre de solution aux enjeux se posant à la ligne de feu.

À titre de troisième exemple de réduction de la complexité, l'étude a permis de dégager un enjeu sur le plan des «erreurs de sélection» des pompes élastomériques à perfusion intraveineuse. Puisque chacun des modèles de pompes permet au liquide de s'écouler à un débit préétabli et qu'il y a de nombreuses options en matière de débit (Figure 4), il est capital que le bon appareil soit choisi par le personnel de pharmacie. Plusieurs incidents dans le cadre desquels le mauvais dispositif avait été rempli et administré ont été signalés dans le cadre du sondage et des études sur le terrain. La recommandation habituelle en vue d'éliminer ce type d'erreur revient à suggérer des politiques exigeant une revérification indépendante du dispositif ou exigeant que les différents modèles de pompes soient conservés dans des zones distinctes de la pharmacie. Bien qu'il s'agisse là de politiques importantes, une stratégie plus efficace en vue de prévenir ce genre d'erreur consiste à uniformiser la pratique dans le but de réduire le nombre de modèles de pompes utilisés dans un centre donné. Moins il $y$ a de modèles de pompes, moins il risque de s'y produire des erreurs de sélection.

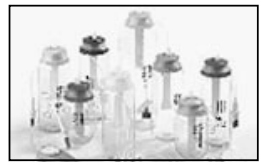

Figure 4.

Divers modèles de pompes

Nous vous encourageons à examiner les élastomériques enjeux se posant à la ligne de feu dans votre à perfusion milieu de travail et à vous demander s'ils pro- intraveineuse de marque Baxter viennent d'une complexité non nécessaire. Il nous faut coopérer à l'élaboration de normes de pratique qui comprennent un moindre nombre de points de décision et moins de confusion.

\section{Conclusions}

La tragédie de la perte d'une patiente en août 2006 a été un puissant catalyseur qui a déclenché toute une série de mesures de première importance. Des changements de taille ont été apportés à la pratique un peu partout dans le pays et nous croyons qu'ils ont permis ou permettront de sauver des vies.

Nos collègues spécialisés en facteurs humains nous rappellent que nous vivrons toujours dans un monde imparfait et que les êtres humains seront toujours faillibles. Si une erreur particulière est susceptible de survenir, on peut être certain qu'elle se produira un jour. Éliminer ces erreurs potentielles avant qu'elles ne surviennent est un pas de géant vers l'amélioration de l'innocuité des traitements. Souvenez-vous qu'il nous est impossible de changer la condition humaine, mais qu'en tant qu'infirmières et administrateurs des soins de santé, nous avons la possibilité de changer les conditions dans lesquelles nous travaillons. Nous devons donc avoir le courage de sortir des rangs et de divulguer nos inquiétudes en matière d'innocuité, nous pouvons collaborer afin de concevoir des améliorations de la sécurité qui fonctionnent pour tous les intervenants et nous pouvons nous efforcer de simplifier les choses sur la ligne de feu en réduisant la complexité en amont et dans l'ensemble du système.

\section{Remerciements}

L'équipe de recherche se composait de représentants d'une vaste gamme d'organismes et de disciplines:

Anthony Easty, $D^{r}$ Anthony Fields, Rachel White, Esther Green, Karen Janes, Andrea Cassano-Piche, Jennifer Jeon, Debbie Chan, Roxanne Dobish, $D^{r}$ Dhali Dhaliwal, $D^{r}$ Eshwar Kumar, Venetia Bourrier, Sylvia Hyland, $D^{r}$ Brent Schacter, $D^{\text {re }}$ Maureen Trudeau, $D^{r}$ Yoo-Joung Koo et Kim Vicente.

Le Comité de l'innocuité des traitements systémiques avait les membres suivants : Susan Walisser, Dre Susan O'Reilly, Denise Budz, Larry Broadfield, Heather MacKenzie, Roxanne Rodgers-Harding, Scott Edwards, Nancy Bestic, Jessica Peters, Melany Leonard, Heather Logan et Jillian Hardy.

Les auteures de l'article et les membres de l'équipe de recherche souhaitent remercier les infirmières de l'ensemble du Canada qui ont participé à l'étude ainsi que l'ACIO/CANO qui a appuyé l'étude. Finalement, les auteures tiennent à souligner le courage de l'équipe du Cross Cancer Institute ainsi que celui de la famille de Denise Melanson.

Reason J. (2000). Human error: Models and management. BMJ, 320(7237), 768-70.

South Dakota Association of Healthcare Organizations. (2009). FMEA Scoring Scale, 2009. Sioux Falls, SD: Author.

United States Department of Veteran Affairs. (2001). The basics of healthcare failure mode and effect analysis. Ann Arbor, MI: Veteran Affairs National Center for Patient Safety.

Vicente, K., \& Christoffersen, K. (2006). The Walkerton E. coli outbreak: A test of Rasmussen's framework for risk management in a dynamic society. Theoretical Issues in Ergonomics Science, $7(2), 93-112$.

Westbrook, J.I., Woods, A., Rob, M.I., Dunsmuir, W.T., \& Day, R.O. (2010). Association of interruptions with an increased risk and severity of medication administration errors. Arch Intern Med, $170(8), 683-90$. modeling problem. Safety Science, 27(2/3), 183-213. 